\title{
Optimality and Conductivity for Water Flow: From Landscapes, to Unsaturated Soils, to Plant Leaves
}

\author{
HUI-HAI LIU \\ Earth Sciences Division \\ Lawrence Berkeley National Laboratory \\ Berkeley, CA 94720, USA \\ hhliu@lbl.gov \\ Phone: (510)486-6452; FAX: (510)486-5686
}




\begin{abstract}
Optimality principles have been widely used in many areas. Based on an optimality principle that any flow field will tend toward a minimum in the energy dissipation rate, this work shows that there exists a unified form of conductivity relationship for three different flow systems: landscapes, unsaturated soils and plant leaves. The conductivity, the ratio of water flux to energy gradient, is a power function of water flux although the power value is system dependent. This relationship indicates that to minimize energy dissipation rate for a whole system, water flow has a small resistance (or a large conductivity) at a location of large water flux. Empirical evidence supports validity of the relationship for landscape and unsaturated soils (under gravity dominated conditions). Numerical simulation results also show that the relationship can capture the key features of hydraulic structure for a plant leaf, although more studies are needed to further confirm its validity. Especially, it is of interest that according to this relationship, hydraulic conductivity for gravity-dominated unsaturated flow, unlike that defined in the classic theories, depends on not only capillary pressure (or saturation), but also the water flux. Use of the optimality principle allows for determining useful results that are applicable to a broad range of areas involving highly non-linear processes and may not be possible to obtain from classic theories describing water flow processes.
\end{abstract}

Key words. Optimality, unsaturated flow, surface hydrology, ecohydrology 


\section{Introduction}

Optimality principles refer to the state of a physical process is controlled by an optimal condition subject to physical and/or resource constraints. For example, Eagleson (2002) demonstrated that under natural conditions and in water-limited areas, vegetation tends to grow under maximum-productivity and unstressed conditions. He called function and forms of vegetation, following the optimality principle, results of "Darwinian expression”. Most recently, Liu (2011a) extended the theory of Eagleson (2002) to a dual-layer system and verified the optimization results with field observations. After studying a variety of natural phenomena characterized by tree-like structures, Bejan (2000) proposed "constructal theory" that states that "for a finite-size open system to persist in time (to survive) it must evolve in such a way that it proves easier and easier access to the currents that flow through it”. Over the past 30 years, the maximum entropy production (MEP) principle has been successfully applied, in a heuristic sense, to the prediction of steady states of a wide range of systems (e.g., Nieven 2010; Kleidon 2009). The MEP principle states that a flow system subject to various flows or gradients will tend towards a steady-state position of maximum thermodynamic entropy production (Nieven 2010). However, the theoretical connections between these optimality principles and the currently existing fundamental laws are not well established. The alternative point of view is that these principles are actually self-standing and do not follow from other known laws (Bejan 2000).

The role of optimality principles in forming complex natural patterns has been recognized for many years in the surface hydrology community (Leopold and Langbein 1962; Howard 1990; Rodriguez-Iturbe et al. 1992; Rinaldo et al. 1992; Liu 2011b). For example, Leopold and Langbein (1962) proposed a maximum entropy principle for studying the formation of landscapes. Rodriguez-Iturbe et al (1992) postulated principles of optimality in energy expenditure at both local and global scales for channel networks.

The importance of optimality principles has also been intuitively recognized in the vadose-zone hydrology community for a long time. For example, it seems to be well known that fingering flow is due to a fact that unsaturated water tends to form flow paths corresponding to the minimized flow resistances. Note that for a given water flux, 
fingering flow always gives lower flow resistance [or higher conductance] compared with uniform flow, because fingering flow paths generally have higher local water saturations that correspond to larger unsaturated conductivities.

Most recently Liu (2011b,c), based on the optimality principle that energy dissipation rate (or flow resistance) is minimized for the entire flow system, demonstrated that conductivity for water flow is a power function of water flux for both landscapes and unsaturated soils (under gravity-dominated conditions). That development is supported by experimental observations and empirical relations. The conductivity herein is defined as water flux divided by the energy gradient along the flux direction. This study extends the work of Liu (2011 b,c) in several aspects. First, the development in Liu (2011b) relied on Manning's equation for describing water flow over landscapes. We will show that Manning's equation is not needed for obtaining a conductivity relationship for overland flow. Second, we will extend the results of Liu (2011c) for unsaturated flow in homogeneous soils to heterogeneous cases. Third, we will demonstrate that the powerfunction relationship between conductivity and water flux may also be applicable to plant leaves. The ultimate objective of this contribution is to show that power-function relationships between conductivity and water flux, resulting from the optimality principle, seems to be common for several very different natural flow systems, although the power value is system dependent.

\section{Steady-state optimal landscape}

Liu (2011b) showed that using an optimality principle leads to a conclusion that conductivity for an optimal landscape is a power function of water flux. However, his work is based on a notion that overland water flow can be described by the well-known Manning's equation. In this section, we demonstrate that the notion is not needed to derive the conductivity relationship. Because this paper describes results for three different systems (landscapes, unsaturated soils and plant leaves), the definitions and physical meanings of a same symbol (denoting a variable or function) may be different in different sections herein unless the same physical meanings are explicitly indicated.

Following Liu (2011b), we consider a landscape involving steady-state water flow and surface evolution processes. This steady-state assumption has been implicitly 
employed in previous studies on topological structures of channel networks (Howard 1990; Rodriguez-Iturbe et al. 1992; Rinaldo et al. 2006). A land surface constantly responds to spatially and temporally variable forcing (such as rainfall). However, it develops average conditions (such as average hydraulic geometry) that are relatively stable on a large time scale (Leopold and Maddock 1953; Molnar and Ramirez 1998). Rinaldo et al. (2006) further indicated that several statistical properties are found to be almost the same for many rivers, irrespective of their age, supporting the steady-state treatment. Along the same line, rainfall is assumed to be steady state and uniform throughout the landscape under consideration. While the model to be discussed can be extended to cases in which land properties (such as those related to soil and vegetation) are spatially heterogeneous, for simplicity we focus on land surfaces with homogeneous properties. Although infiltration processes occur during a rainfall event, the resulting infiltration flux generally corresponds to a relatively small portion of the rainfall flux during periods of heavy rainfall that are relevant to landscape evolution processes. Therefore, infiltration is ignored in this study.

Based on the above simplifications, coupled water-flow (over a land surface) and surface-elevation equations can be derived from the principle that global energy expenditure rate is at the minimum. From the water mass (volume) conservation, the steady-state water flow equation is given by

$$
\frac{\partial q_{x}}{\partial x}+\frac{\partial q_{y}}{\partial y}=Q
$$

where $x$ and $y$ are two horizontal coordinate axes, $q_{x}$ and $q_{y}$ are water fluxes (water velocity multiplied by water depth) along $x$ and $y$ directions, respectively, and $Q$ is the rainfall rate.

Accordingly, the energy expenditure rate for a unit land-surface area, $\Delta_{E}$, can be expressed as (based on energy conservation)

$$
\Delta_{E}=\frac{\partial\left(q_{x} E\right)}{\partial x}+\frac{\partial\left(q_{y} E\right)}{\partial y}-Q E
$$

The above equation simply states that for a given unit area, the energy expenditure rate at that location is equal to the energy carried by water flowing into the area minus the energy carried by water flowing out of the area. The rainfall is assumed to have the same 
energy as water at the location where the rain falls. The energy, $E$, (a function of $x$ and $y$ ) represents the total energy including both potential (corresponding to elevation $z$ ) and kinetic energy:

$$
E=z+\frac{v^{2}}{2 g}
$$

where $g$ is gravitational acceleration and $v$ is water velocity. Note that the second term is generally small and has been ignored in some previous studies (e.g., Howard 1990; Rinaldo et al. 2006). For completeness, this term is included here.

A combination of Equations (1) and (2) yields

$$
\Delta_{E}=q_{x} \frac{\partial E}{\partial x}+q_{y} \frac{\partial E}{\partial y}
$$

The water flux is considered to be given by

$$
\begin{aligned}
& q_{x}=-K\left(h, S_{*}\right) \frac{\partial E}{\partial x} \\
& q_{y}=-K\left(h, S_{*}\right) \frac{\partial E}{\partial y}
\end{aligned}
$$

where

$$
S_{*}=S^{2}=\left(\frac{\partial E}{\partial x}\right)^{2}+\left(\frac{\partial E}{\partial y}\right)^{2}
$$

In Equations (5a) and (5b), K(h, $\left.S_{*}\right)$ is conductivity and $h(\mathrm{~m})$ is water depth. Note that in Liu (2011b), the specific formulation of conductivity is given based on the Manning's equation. In this study, we assume $h$ to be a function of local slope $S$ only (Gupta and Waymire 1989). Many studies indicate that on average a number of hydraulic parameters can be considered as functions of local slope (Leopold and Maddock 1953). In this case, $\mathrm{K}$ is the function of $\mathrm{S}_{*}$ only.

When we combine Equations (4) and (5), the global energy expenditure rate throughout domain $\Omega$ is given by

$$
\iint_{\Omega} \Delta_{E} d x d y=\iint_{\Omega}\left(-K S_{*}\right) d x d y
$$

The optimality principle in our problem is to minimize the absolute value of the above integral. To do so, we employ the calculus of variations that seeks optimal 
(stationary) solutions to a functional (a function of functions) by identifying unknown functions (Weinstock 1974). For example, the former corresponds to the integral defined in Equation (6) and the latter to land-surface elevation distribution $z(x, y)$.

Furthermore, we employ the following constraint for the optimization problem (Liu 2011b):

$$
\iint_{\Omega} E d x d y=C
$$

where $C$ is a constant. Since $E$ is mainly composed of potential energy $z$, the above equation essentially states that the average elevation throughout the model domain (or total volume of the landscape under consideration) remains unchanged, which is consistent with the steady-state assumption made in this study. It should be emphasized that the optimality principle corresponds to minimization of the global energy expenditure rate, not the total energy within the model domain. Under steady state conditions, the global energy expenditure is equal to the difference between the latter and energy carried by water flowing out of the system.

Based on Equations (5), (6) and (7), the Lagrangian for the given problem is given by $L=-K S_{*}+\lambda_{1}^{*}\left[S_{*}-\left(\frac{\partial E}{\partial x}\right)^{2}-\left(\frac{\partial E}{\partial y}\right)^{2}\right]+\lambda_{2}{ }^{*}[E-C]$

Note that the first term is from Equation (6) and other terms are constraints from Equations (5) and (7). Use of these constraint terms allows considering related functions to be independent when determining the optimal solution to Equation (8). The Lagrange multipliers $\lambda_{1}{ }^{*}$ and $\lambda_{2}{ }^{*}$ are a function of location and a constant, respectively. The last term on the right hand side of Equation (8) corresponds to the constraint defined in Equation (7). Note that the constraint related to water flow, Equation (1), is not included in Equation (8), but will be handled later for mathematical convenience.

The following Euler-Lagrange equation is used to determine an unknown function w associated with $L$ to minimize the integral defined in Equation (8) (Weinstock 1974; Pike 2001):

$$
\frac{\partial L}{\partial w}-\frac{\partial}{\partial x}\left(\frac{\partial L}{\partial w_{x}}\right)-\frac{\partial}{\partial y}\left(\frac{\partial L}{\partial w_{y}}\right)=0
$$


where $w_{x}$ and $w_{y}$ are partial derivatives with respect to $x$ and $y$, respectively. In this study, $w$ corresponds to $S *$ and $E$, respectively.

Applying the Euler-Lagrange Equation (9) to $S *$ in (8) gives

$\lambda_{1}^{*}=\frac{d\left(K S_{*}\right)}{d S_{*}}$

Applying the Euler-Lagrange Equation (9) to E yields

$\lambda_{2}^{*}+\frac{\partial}{\partial x}\left(2 \lambda_{1}^{*} \frac{\partial E}{\partial x}\right)+\frac{\partial}{\partial y}\left(2 \lambda_{1}^{*} \frac{\partial E}{\partial y}\right)=0$

For the optimization results to be physically valid, they must satisfy the water flow equation (1). A direct comparison between Equations (1) and (11) and consideration of (5) reveal that Equations (1) and (11) are identical under the following conditions

$\lambda_{1}^{*}=\left(\frac{\lambda_{2}^{*}}{2 Q}\right) K$

Combining Equations (10) and (12), we can obtain

$K \propto S_{*}{ }^{-1+\frac{\lambda_{2}^{*}}{2 Q}}=S^{-2+\frac{\lambda_{2}^{*}}{Q}}$

From Equations (5) and (13), water flux $q$ and local slope $S$ has the following relationship:

$q \propto S^{-1+\frac{\lambda_{2} *}{Q}}$

where

$q=\sqrt{q_{x}^{2}+q_{y}^{2}}$

The power-function relationship between water flux (or discharge) and local slope has been intensively investigated and validated in the literature (Rodriguez-Iturbe et al. 1992; Rinaldo et al. 2006; Banavar et al. 2001). Also note that the power value in the powerfunction relationship varies with different site conditions. However, previous studies (Leopold and Maddock 1953; Rodriguez-Iturbe et al. 1992; Rinaldo et al. 2006; Banavar et al. 2001) indicate that the averaged power value is about -2 in (14), suggesting that $\frac{\lambda_{2} *}{Q}$ is close to -1 in an average sense. 
Equations (13) and (14) lead to a relationship between the flow conductance $K$ (flux divided by energy gradient) and flux:

$K \propto q^{\frac{2-\frac{\lambda_{2} *}{Q}}{1-\frac{\lambda_{2} *}{Q}}}$

When $\frac{\lambda_{2} *}{Q}$ is close to -1 , the power value in the above equation is about 1.5 . The equation indicates that under optimal conditions, locations where a relatively large water flux occurs correspond to a relatively small resistance (or large conductance). As demonstrated in the sections to follow, that flow conductance is a power function of water flux seems to be a common rule under optimal flow conditions.

\section{Unsaturated flow in porous media}

For a homogeneious soil, Liu (2011c) reported that unsaturated conductivity, similar to Equation (16), is a power function of water flux under gravity-dominant and optimal flow conditions. This section will show that the same power-function relationship can be applied to heterogeneous soils as well.

We consider a relatively simple, steady state unsaturated flow system associated with a heterogeneous and isotropic porous medium. From the water mass (volume) conservation, the steady-state water flow equation is given by

$$
\frac{\partial q_{x}}{\partial x}+\frac{\partial q_{y}}{\partial y}+\frac{\partial q_{z}}{\partial z}=0
$$

where $x$ and $y$ are two horizontal coordinate axes, $\mathrm{z}$ is the vertical axis, and $q_{x}, q_{y}$ and $\mathrm{q}_{\mathrm{z}}$ are volumetric fluxes of water along $x, y$ and $z$ directions, respectively.

We use $E$ (a function of $x, y$ and $z$ ) to represent the total energy including both potential (corresponding to elevation $z$ ) and (capillary) pressure energy:

$$
E=z+\frac{P}{\rho g}=z+h
$$

where $g$ is gravitational acceleration, $\mathrm{P}$ is capillary pressure, $\rho$ is water density, and $\mathrm{h}$ is capillary pressure head. Accordingly, the energy expenditure rate for a unit control volume, $\Delta_{E}$, can be expressed as 
$\Delta_{E}=\frac{\partial\left(q_{x} E\right)}{\partial x}+\frac{\partial\left(q_{y} E\right)}{\partial y}+\frac{\partial\left(q_{z} E\right)}{\partial z}$

The above equation simply states that for a given unit volume, the energy expenditure rate at that location is equal to the energy carried by water flowing into the volume minus the energy carried by water flowing out of the volume.

A combination of Equations (17) and (19) yields

$\Delta E=q_{x} \frac{\partial E}{\partial x}+q_{y} \frac{\partial E}{\partial y}+q_{z} \frac{\partial E}{\partial z}$

Throughout this development, Darcy's law is assumed to applied to unsaturated flow:

$q_{x}=-K \frac{\partial E}{\partial x}$

$q_{y}=-K \frac{\partial E}{\partial y}$

$q_{z}=-K \frac{\partial E}{\partial z}$

where $\mathrm{K}$ is hydraulic conductivity and given by

$K=K_{s}(x, y, z) k_{r}\left(h, S_{*}\right)$

$S_{*}=\left(\frac{\partial E}{\partial x}\right)^{2}+\left(\frac{\partial E}{\partial y}\right)^{2}+\left(\frac{\partial E}{\partial z}\right)^{2}$

In Equation (5d), $K_{s}$ is the saturated hydraulic conductivity that is a function of location. The relative permeability $k_{r}$ is assumed to be a function of both capillary pressure head (h) and the square of the energy gradient $\left(S_{*}\right)$. To be able to get closed-form results, we further assume that the function form of relative permeability is independent of location. Assuming $\mathrm{k}_{\mathrm{r}}$ to be a function of water flux is equivalent to assuming it to be a function of energy gradient, because water flux, energy gradient and K are related through Darcy's law. The functional form of $\mathrm{k}_{\mathrm{r}}$ [Equation (21d)] is a subject of study in this work. Note that our theory here is developed for a macroscopic scale that may include a number of fingering or preferential flow paths. Local scale refers to the continuum scale within each finger. Unsaturated flow process on a local scale is mainly controlled by pore-scale physics. We also need to emphasize that assuming $\mathrm{k}_{\mathrm{r}}$ to be a function of $\mathrm{S}_{*}$ on 
macroscopic scale does not exclude the possibility that $\mathrm{k}_{\mathrm{r}}$ may have nothing to do with $\mathrm{S}_{*}$ in our final results. In this case, the mathematically derived function form of $\mathrm{k}_{\mathrm{r}}$ would not include $\mathrm{S}_{*}$ as an independent variable.

When we combine Equations (20) and (21), the global energy expenditure rate is the same as Equation (6). The corresponding Lagrangian for the given problem is given by

$$
L=-K S_{*}+\lambda_{1}\left[S_{*}-\left(\frac{\partial E}{\partial x}\right)^{2}-\left(\frac{\partial E}{\partial y}\right)^{2}-\left(\frac{\partial E}{\partial z}\right)^{2}\right]
$$

Replacing $w$ with $\mathrm{S} *$ in the Euler-Lagrangian Equation (8) yields

$\lambda_{1}=\frac{\partial\left(K S_{*}\right)}{\partial S_{*}}$

Replacing $w$ with $h$ (or E) in Equation (22) and using (23) and the continuity equation, we have

$\frac{\partial\left(\frac{\partial K}{\partial\left(\log S_{*}\right)} \frac{\partial E}{\partial x}\right)}{\partial x}+\frac{\partial\left(\frac{\partial K}{\partial\left(\log S_{*}\right)} \frac{\partial E}{\partial y}\right)}{\partial y}+\frac{\partial\left(\frac{\partial K}{\partial\left(\log S_{*}\right)} \frac{\partial E}{\partial z}\right)}{\partial z}=\frac{S_{*}}{2} \frac{\partial K}{\partial h}$

In general, it is difficult to obtain an analytical solution to the above equation. However, for some special case in which the term on the right hand side is small compared to other terms, a closed-form solution can be obtained. This is true for a gravity-dominant flow (Liu 2011b). A comparison between Equation (24) (without the term on the right hand side) with the continuity equation (Equations (17) and (21)) yields

$\frac{\partial K}{\partial\left(\log S_{*}\right)}=A K$

where $A$ is a constant.

To get practically useful results, we consider $\mathrm{K}$ to be further expressed by

$$
K=K_{s}(x, y, z) k_{r}\left(h, S_{*}\right)=K_{s}(x, y, z) f(h) g\left(S_{*}\right)
$$

Substituting (26) into (25) results in (for a given location)

$$
g\left(S_{*}\right) \propto S_{*}{ }^{A}
$$

Based on Darcy's law, (27) can be rewritten as

$$
g\left(S_{*}\right) \propto\left(\frac{|q|}{K}\right)^{A / 2}
$$


where $|q|$ is the magnitude of water flux given by

$|q|=\left[q_{x}{ }^{2}+q_{y}{ }^{2}+q_{z}{ }^{2}\right]^{1 / 2}$

Combining (28) and (26) gives our final conductivity relationship as follows

$K=K_{s}(x, y, z) F(h)\left(\frac{|q|}{K_{s}}\right)^{a}$

where $a$ is a constant exponent for the conductivity-flux power law. Equation (30-1) may also be rewritten as

$k_{r}\left(h, S_{*}\right)=F(h)\left(\frac{|q|}{K_{s}}\right)^{a}$

There may be different interpretations of Equation (30). One interpretation is that $\mathrm{F}(\mathrm{h})$ is the local-scale relative permeability within the fingering-flow zone and that the power function of flux in the equation represents the fraction of fingering flow zone in an area normal to water flux direction. The validity of (30) for homogeneous soils was demonstrated in Liu (2011c) with both laboratory-experimental observations of vertical fingering flow (Wang et al. 1998) and field observations (Sheng et al. 2009). Based on these observations, Liu (2011c) also speculated that parameter $a$ may have a universal value of 0.5 , which, however, needs further verification.

Equation (30) clearly shows that for a gravity-dominated unsaturated flow, relative permeability is not only a function of water potential (or saturation), but also a power function of water flux. Treating relative permeability as a function of water potential (or saturation) only has been widely used in the literature of vadose zone hydrology. This needs to be revisited, because the treatment is based on a local-equilibrium assumption that capillary pressure is uniform within a representative elementary volume (REV). This is obviously violated at a large scale by the existence of fingering flow. The powerfunction term in (30) largely reflects the self-organization of flow patterns driven by the minimization of total flow resistance. Finally, it is important to note that the classic unsaturated-flow theory is applicable to capillarity-dominated cases (in which local equilibrium holds) while the current work focuses on unsaturated flow under gravity- 
dominated conditions. Whether the optimality principle can be used to develop a general theory (that includes the both cases as two special ones) deserves further research.

Application of (30) to model unsaturated flow can follow the procedure used in the socalled active region model (ARM) (Liu et al. 1998, 2003, 2005; Sheng et al. 2009). ARM divides the flow domain into two parts: active and inactive. Water flow occurs within the active region only, and inactive region is simply bypassed. The formulation to calculate the volumetric fraction of the active region can be related to (30), as shown in Liu (2011b). Specifically, F(h) and power-function term in (30) can be considered as the relative permeability within the active region and the volumetric fraction of that region, respectively. Also note that the derivation of (30) does not consider the physics of water flow at pore scale. To get physically valid results, the relative permeability calculated from (30) needs to be limited by a upper limit of $S_{e}$ and a lower limit of $k_{r}{ }^{*}\left(S_{e}\right)$, where $S_{e}$ is the effective saturation defined as water volume (excluding residual water) divided by porosity (excluding pore space occupied by residual water) and $\mathrm{k}_{\mathrm{r}}{ }^{*}\left(\mathrm{~S}_{\mathrm{e}}\right)$ is the local relative permeability at saturation $S_{\mathrm{e}}$. The rational for these limits and detailed numerical procedure of ARM can be found in Liu et al. (2003) and Sheng et al. (2009).

\section{Optimal hydraulic structure for a plant leaf}

In the above two sections, we have shown that conductivities for water flow over landscapes and within unsaturated soils (under gravity-dominated conditions) are power functions of the relevant water flux. In this section, we further demonstrate that the same form of conductivity relationship may also be applicable to hydraulic structure for a plant leaf.

For simplicity, we assume that a plant leaf can be treated as a two-dimensional saturated porous medium with a uniform evaporation rate. The flow within a plant leaf is in steady state. The similar assumption was also made by Bejan (2000). Under these conditions, the continuity equation (1) and energy expenditure equation (2) for the landscapes can be used for a plant leaf, except that $Q$ in (1) and energy E in (2) correspond to evaporation rate and pressure head, respectively, in this section.

The water-flux is described by Darcy's law: 
$q_{x}=-K \frac{\partial E}{\partial x}$

$q_{y}=-K \frac{\partial E}{\partial y}$

where $\mathrm{K}$ is hydraulic conductivity for a plant leaf in this section and a function of location. Again, we use equation (5c) to denote the square of energy gradient. The major purpose of this section is to find the spatial distribution of $\mathrm{K}$ under optimal flow conditions. For this optimization problem, we further impose a constraint given by

$\iint K d x d y=C_{*}$

The above constraint simply implies that we are seeking an optimized spatial distribution of $\mathrm{K}$ for a given average $\mathrm{K}$ value over a whole plant leaf which is needed to bound final results.

Following the procedure to derive (8), the Lagrangian for the given problem is given by

$$
\begin{aligned}
& L=-K S_{*}+\lambda_{1}\left[-Q+\frac{\partial q_{x}}{\partial x}+\frac{\partial q_{y}}{\partial y}\right]+\lambda_{2}\left[S_{*}-\left(\frac{\partial E}{\partial x}\right)^{2}-\left(\frac{\partial E}{\partial y}\right)^{2}\right] \\
& +\lambda_{3}\left[q_{x}+K \frac{\partial E}{\partial x}\right]+\lambda_{4}\left[q_{y}+K \frac{\partial E}{\partial y}\right]+\lambda_{5}\left(K-C_{*}\right)
\end{aligned}
$$

Note that the first term is from Equation (6) and other terms are constraints from Equations (1), (5c), (31) and (32).

Again, Euler-Lagrange equation (9) is used to obtain the solution to minimizing (33). Replacing $w$ with $K$ in Equation (9) and using the definition of $S_{*}$ (Equation (5c)), we obtain

$$
\left(\lambda_{3}-\frac{\partial E}{\partial x}\right) \frac{\partial E}{\partial x}+\left(\lambda_{4}-\frac{\partial E}{\partial y}\right) \frac{\partial E}{\partial y}=\lambda_{5}
$$

Replacing $w$ with $q_{x}$ and $q_{y}$, respectively, in Equation (9) yields

$$
\lambda_{3}=\frac{\partial \lambda_{1}}{\partial x}
$$


$\lambda_{4}=\frac{\partial \lambda_{1}}{\partial y}$

Replacing $w$ with $S *$ in Equation (9), we have

$\lambda_{2}=K$

Replacing $w$ with $E$ in Equation (9), letting $\lambda=\lambda_{1}-E$, and using Equations (1), (35) and (36), we obtain

$\frac{\partial\left[K \frac{\partial \lambda}{\partial x}\right]}{\partial x}+\frac{\partial\left[K \frac{\partial \lambda}{\partial y}\right]}{\partial y}=-Q$

By definition of $\lambda$, Equation (34) can be rewritten as

$\frac{\partial \lambda}{\partial x} \frac{\partial E}{\partial x}+\frac{\partial \lambda}{\partial y} \frac{\partial E}{\partial y}=\lambda_{5}$

Combining Equations (1) and (31) gives a new form of water flow equation:

$\frac{\partial\left[K \frac{\partial E}{\partial x}\right]}{\partial x}+\frac{\partial\left[K \frac{\partial E}{\partial y}\right]}{\partial y}=-Q$

A combination of Equations (32) and (37) to (39) corresponds to the minimization of the absolute value of global energy expenditure rate in Equation (33). Comparing (37) with (39), one obvious solution to these equations is

$\lambda=E$

In this case, Equations (37) and (39) becomes identical. Combining Equations (38), (40) and (31) and noting that $\lambda_{5}$ is constant, we have

$K \propto q$

where $q$ is the magnitude of water flux and defined in Equation (15). Again, conductivity and flux exhibit a power-function relationship. (Linear function is a special case of the power function.)

In the previous two sections, we have demonstrated that the derived conductivity relationships are supported by observations or empirical relations for landscapes and 
unsaturated soils, respectively. However, to our best knowledge, a data set for detailed water flow process across a plant leaf does not seem to be available in the literature. To examine the reasonableness of Equation (41), we numerically solve relevant equations to obtain the spatial distribution of $\mathrm{K}$ for a plant leaf. Note that the shape and hydraulic structures of a plant leaf are likely dependent on biological processes, which are not considered. Thus, we do not expect our simulation to generate very realistic hydraulic structures (that are characterized by the thickness distribution of the leave and/or its blade pattern). Instead, our focus here is on whether our model results can capture key features of plant-leaf hydraulic structure.

We solve Equations (39), (41), (32) to obtain an optimal K distribution. Because transient problem is relatively easy to solve, we replace (39) by a transient equation:

$$
\frac{\partial\left[K \frac{\partial E}{\partial x}\right]}{\partial x}+\frac{\partial\left[K \frac{\partial E}{\partial y}\right]}{\partial y}+Q=\frac{\partial E}{\partial t}
$$

where $t$ is time. The steady-state solution to (42) will be the same as that for (39). In our simulations, we set $\mathrm{Q}=\mathrm{C}_{*}=1$ and use the uniform numerical grids. Equation (42) is a non-linear diffusion equation and numerically solved using an explicit finite-difference method. To obtain stable solution, the time step is limited by (Konikow and Bredehoeft, 1984):

$\Delta t \leq \frac{(\Delta x)^{2}}{2 K_{\max }}$

where $\mathrm{K}_{\max }$ is the maximum $\mathrm{K}$ value within the leaf and $\Delta x$ is the size of a numerical gridblock.

An iterative procedure is employed to obtain the optimal $\mathrm{K}$ distribution. For an assumed K distribution and given boundary conditions, we solve (42) to obtain the corresponding steady-state $\mathrm{E}$ distribution based on which we can calculate water flux using (31) at each location. Then, we use the new flux distribution and Equations (32) and (41) to update the $\mathrm{K}$ distribution. We solve (42) again with the updated $\mathrm{K}$ distribution. This procedure is repeated until the $\mathrm{K}$ distributions are essentially the same between two iterations. 


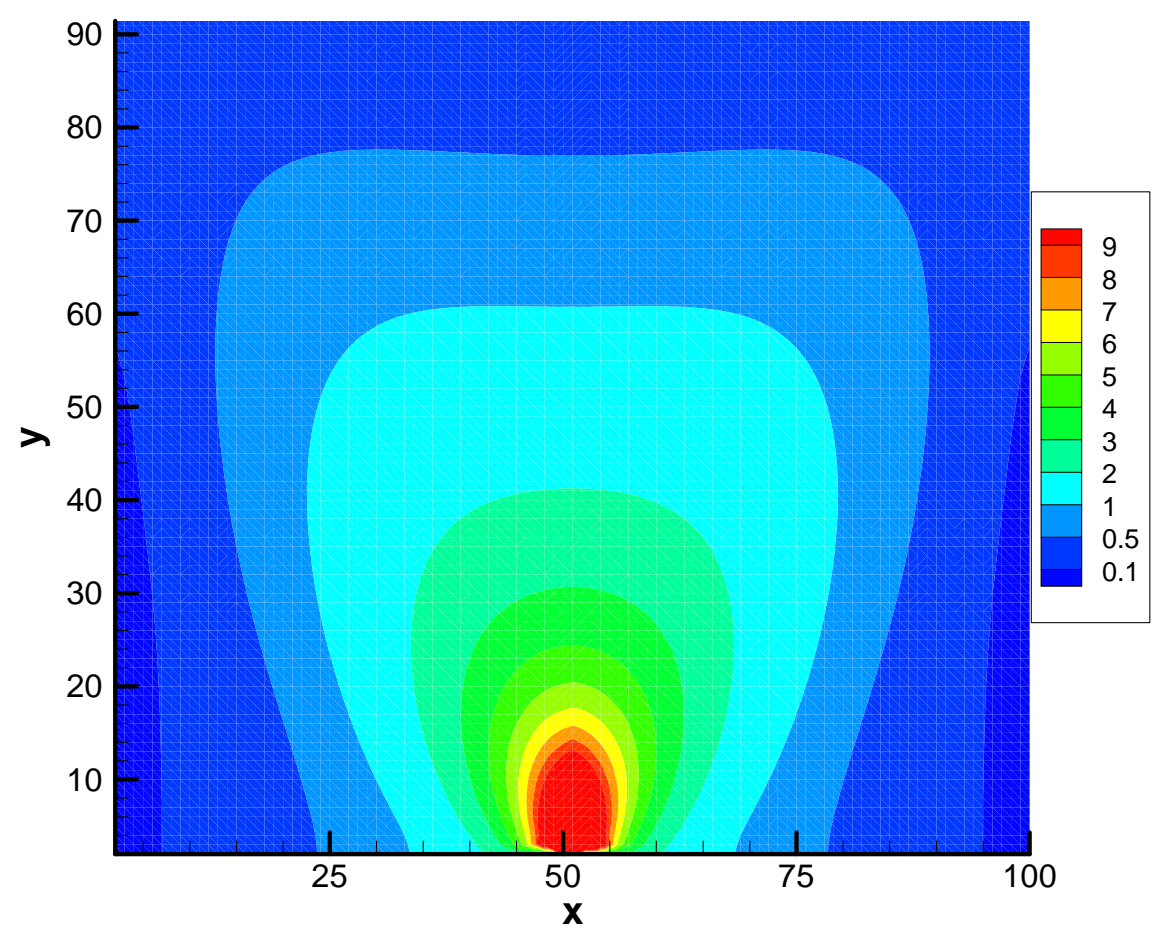

Fig 1. Simulated conductivity distribution of a plant leaf.

For simplicity, we use a rectangle to represent a leaf, which, of course, is a rough approximation considering that plant leaves take a variety of shapes. As shown in Figure 1, non-flow boundary conditions are imposed to the four sides. Evaporation rate is uniformly distributed across the leaf. We also assume the leaf to be connected with a stem at the location (with $\mathrm{x}=50$ and $\mathrm{y}=3$ ) where the pressure $(\mathrm{E})$ is fixed to be zero. All the water evaporated from the leaf comes from that location. A $100 \times 100$ grid system is used in our simulation.

Figure 1 shows a simulated $\mathrm{K}$ distribution that has two interesting features. First, $\mathrm{K}$ keeps increasing from leaf edges to the source location where the leaf is connected with the stem. This is because water flux increases near the source. Second, K changes gradually and its distribution does not show obvious structures. Note that changes in $\mathrm{K}$ can be reflected by the change in thickness of a real leaf. Leaves without obvious 
structures can be found in some desert plants including big-leaf mistletoe, desert cabbage, succulent, and desert agave.

Ginkgo leaves also have similar K distributions. They consist of many small continuous blades that serve as flow paths between the source and other parts of the leaves. Since these blades are densely distributed, the leaves are viewed as structureless. In this case, K value can be considered to be proportional to the blade density. Similar to Figure 1, the leaves have much higher density (and larger blade sizes) near the source.

A common plant leaf has a hydraulic structure characterized by the corresponding blade pattern on it. Blades have a small flow resistance or large conductivity. However, Figure 1 does not show this structure. This may be because we do not consider some biological constraints in the above simulation. One important biological constraint is that pressure cannot be too low such that the corresponding plant is deeply stressed (Kramer and Boyer 1995). This may explain why leaves of some desert or dry-area plants have K distributions like that in Figure 1, because these plants can tolerate high stresses. To further examine this hypothesis, we limit the magnitude of pressure (E) within a leaf to a give value, $\mathrm{E}_{\max }$, using the following transformation:

$E=E_{\text {max }}\left(1-e^{-T}\right)$

where $\mathrm{T}$ is function of locations. We replace $\mathrm{E}$ in (39) with $\mathrm{T}$ by inserting (44) into (33), and then determine $\mathrm{T}$ distributions following the same procedure to determine $\mathrm{E}$ distributions. It can be mathematically shown that inclusion of the constraint (44) in the Lagrangian defined in (33) does not change the conductivity relationship (Equation (41)). Since Equation (44) introduces additional non-linearity, the numerical procedure becomes more computationally intensive. 


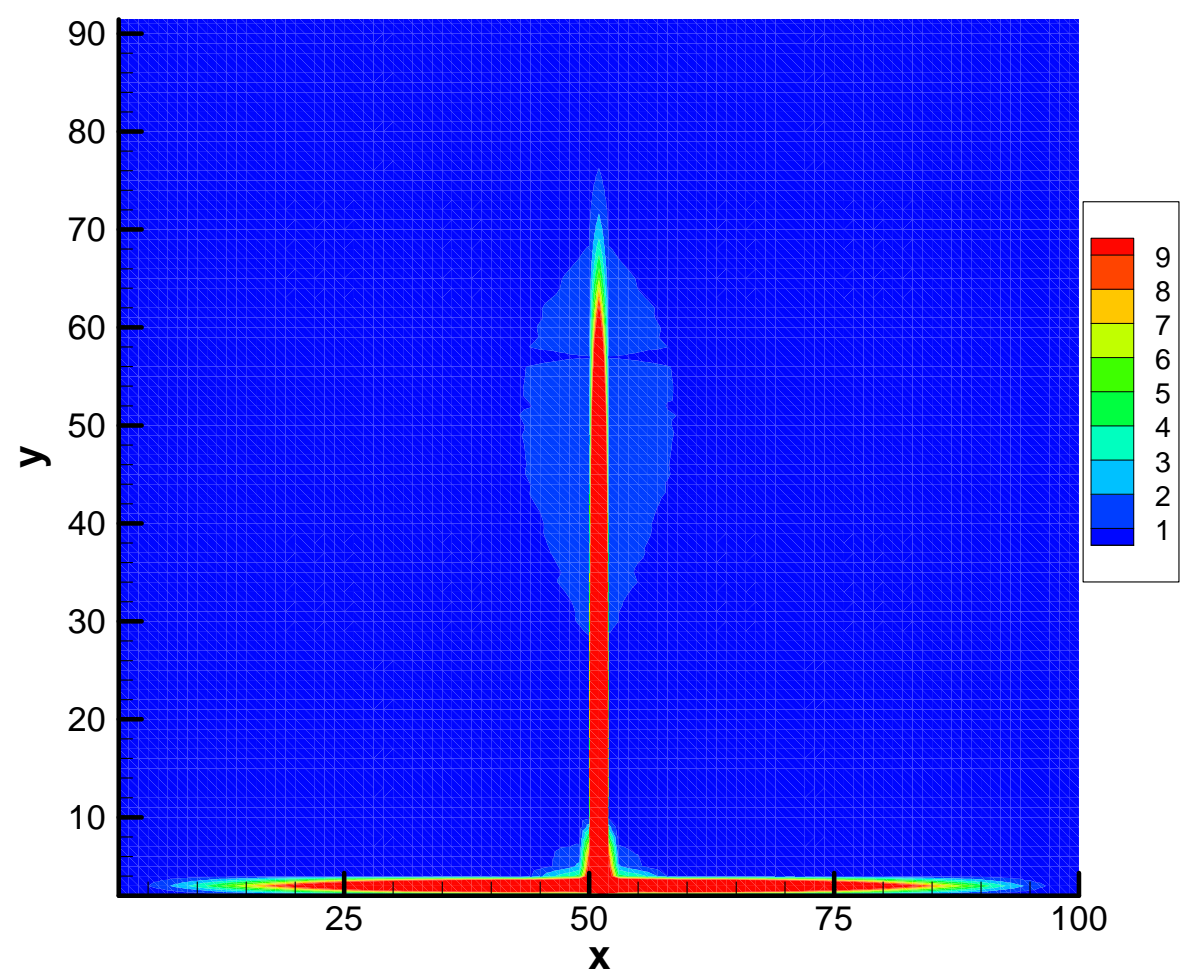

Fig 2. Simulated conductivity distribution of a plant leaf with the additional constraint defined in Equation (44).

Figure 2 shows simulation results with the new constraint. The ratio of maximum pressure in this simulation to that associated with Figure 1 is 0.06 . As expected, some high-conductivity features occur in the middle and at the bottom of the leaf and seem to be consistent with large blades on a real leaf in the general sense. However, relatively small blades, often observed in a real leaf, are not generated in Figure 2. There may be several reasons for that. As previously indicated, formation of a blade pattern may be subject to many biological constraints that are not carefully considered herein. It may be possible that use of a lower pressure ratio generates a blade pattern with more blades at different scales. However, we were not able to get numerically convergent results for cases with much lower pressure ratios as a result of increasing non-linearity. A more advanced numerical approach is needed for future studies. Nevertheless, simulation results given in Figures 1 and 2 seem to be able to capture major hydraulic-structure 
features of leaves for different plants, although the validity of the conductivity relationship needs to be further confirmed by experimental observations.

\section{Discussions}

As discussed in the introduction section, optimality principles have been widely used in different areas. However, most of these principles are not established based on classical mechanics and thermodynamics, and therefore one generally faces two questions when applying them: Are the optimality principles valid for the problems under consideration? Are these principles independent of the currently existing laws?

This study is based on a particular optimality principle that energy dissipation rate (or flow resistance) is minimized for the entire flow system. This allows us to unify the conductivity relationships for three seemingly different systems: landscape, unsaturated soil and plant leaf. (This may partially explain why flow patterns in these three systems are similar.) There is a considerable amount of experimental evidence to support the validly of our results, although more studies are needed to further confirm the result for plant leaves. This in turn demonstrates the validity of the optimality principle that we have employed. It is of interest to note that all the systems under consideration have the similar feature that water flow processes and formation of flow patterns are strongly coupled in highly non-linear ways. For example, landscapes are formed by water-flowinduced erosion and fingering pattern formation in unsaturated soils are also closely related to non-linear water flow processes. In the other words, the existence of strong feed backs between flow pattern formation and the flow process itself may likely be the underlying reason that the optimality principle is valid for these diverse circumstances. Whether the optimality principle (that energy dissipation rate is minimized for the entire flow system) is independent of the currently existing laws is a very important question. If it was not independent, there would be no real scientific need to investigate flow processes with the principle, because results from the principle are equivalent to those obtained from the existing physical laws. Again, there are no conclusive answers to this question. However, we tend to agree with Bejan (2000) on that the optimality principle is actually self-standing and does not follow from other known laws. This is simply because 
our conductivity relationships obtained from the optimality principle, to the best of our knowledge, cannot be obtained from the currently existing physical laws.

While there are a number of studies on applications of the optimality in the literature, our study is unique in revealing that conductivity is a power function of water flux in three water-flow systems. This interesting finding has several important implications. First, it makes sense within the context of resource (or conductance) allocation. The power-function relationship with a positive power value always gives a small flow resistance at a location with a large flux, such that flow in the whole system is the most efficient. This allocation strategy is consistent with our daily-life experience. For example, in a highway system, locations with high traffic flux are generally wider or have larger conductivities. Second, while complex partial differential equations are involved in our derivation procedure, the form of our results (power function) is very simple. This form likely has something to do with a fractal pattern that has been observed and studied intensively in the literature (e.g., Feder 1988). In general, a fractal has many features that can be characterized by power functions. A detailed exploration of linkage between our finding and fractals is beyond the scope of this paper and left to future investigations. Third, one grand challenge facing us in the area of hydrogeology is the need to develop physical laws for large-scale multiphase-flow problems. At a local-scale, fluid distribution is mainly controlled by capillarity and not sensitive to flow conditions. That is why relative permeability at local scale can be successfully described as a function of saturation (or capillary pressure) only. At a large scale, this is not the case anymore, although local-scale relationship has been widely used at large scale because alternatives are unavailable. It is fair to say that as a result of the high non-linearity involved, how to model large-scale multiphase flow is an issue that has not been resolved at a fundamental level. Our results (Eq. (30)) suggests that functional forms of large-scale relationships to describe multiphase flow are very likely different from their counterparts at local scales, which cannot be resolved from upscaling based on the same functional forms as those at local scales. It is our hope that the optimality approach may provide an important way to obtain such large-scale relationships. 


\section{Concluding remarks}

Optimality principles have been widely used in many areas. Based on an optimality principle that the water-flow energy dissipation rate is minimal, this work shows that there exists a unified form of conductivity relationship for three different flow systems: landscapes, unsaturated soils and plant leaves. The conductivity, the ratio of water flux to energy gradient, is a power function of water flux although the power value is system dependent. This relationship indicates that to minimize energy dissipation for a whole system, water flow has a small resistance (or a large conductivity) at a location of large water flux. Empirical evidence supports the relationship for landscape and unsaturated soils (under gravity dominated conditions). Numerical simulation results also show that the relationship can capture the key features of hydraulic structure for a plant leaf, although more studies are needed to further confirm its validity. Especially, according to this relationship, hydraulic conductivity for gravity-dominated unsaturated flow, unlike that defined in the classic theories, depends on not only capillary pressure (or saturation), but also the water flux. Use of the optimality principle allows for determining useful results that are applicable to a broad range of areas involving highly non-linear processes and that may not be possible to obtain from classic theories describing water flow processes.

Finally, we need to emphasize that this study represents the first step to develop a unified theoretical framework to describe flow processes in three different systems. Since the optimality principle may hold the key to dealing with complex processes related to water flow, more work is highly desirable along the line discussed here, including the further confirmation of results from this study, exploration of the usefulness of our results in other flow systems, and application of the results to modeling flow processes in the relevant systems. 


\section{Acknowledgement}

We are indebted to Drs. Jim Houseworth and Dan Hawkes at Lawrence Berkeley National Laboratory for their critical and careful review of a preliminary version of this manuscript. This work was supported by the U.S. Department of Energy (DOE), under DOE Contract No. DE-AC02-05CH11231. 


\section{References}

Banavar, J.R., Colaiori, F., Flammini, A., Maritan A., Rinaldo, A.: Scaling, optimality and landscape evolution. Journal of Statistical Physics, 104 (1/2), 1-48 (2001)

Bejan, A.: Shape and structure, from engineering to nature. Cambridge University Press (1992)

Eagleson, P.S.: Ecohydrology: Darwinian expression of vegetation form and function. Cambridge University Press, New York (2002)

Feder, J.: Fractals. Plenum, New York (1998)

Gupta, V. K., Waymire, E.: Statistical self-similarity in river networks parameterized by elevation. Water Resour. Res. (1989) doi:10.1029/WR025i003p00463

Howard, A.D.: Theoretical model of optimal drainage networks. Water Resour. Res. 26(9), 2107-2117 (1990)

Kleidon, A.: Nonequilibrium thermodynamics and maximum entropy production in the Earth system: Application and implications, Naturwissenschaften 96, 653-677 (2009)

Konikow, L.F., Bredehoeft, J.D.: Computer model of two-dimensional solute transport and dispersion in ground water. U.S. Geological Survey, Techniques of Water Resources Investigations, Book 7, Chapter C2 (1984)

Kramer, P.J., Boyer, J.S.: Water relations of plants and soils. Academic Press, New York (1995)

Leopold, L.B., Langbein: The concept of entropy in landscape evolution. U.S. Geol. Surv. Prof. Pap. 500-A (1962).

Leopold, L.B., Maddock, T.: The hydraulic geometry of stream channels and some physiographic implications, U.S. Geol. Surv. Prof. Pap. 252 (1953)

Liu, H.H.: Impact of climate change on groundwater recharge in dry area: An ecohydrology approach. Journal of Hydrology 407, 175-183. doi:10.1016/j.jhydrol.2011.07.024 (2011a)

Liu, H.H.: A note on equations for steady-state optimal landscapes. Geophysical Research Letter, 38, L10402, DOI: 10.1029/2011GL047619 (2011b)

Liu, H. H.: A conductivity relationship for steady-state unsaturated flow processes under optimal flow conditions. Vadose Zone J. 10(2), 736-740, doi:10.2136/vzj2010.0118 (2011c)

Liu H H, Zhang, R.D., Bodvarsson, G.S.: An active region model for capturing fractal flow patterns in unsaturated soils: Model development. J Contam Hydrol 80(1-2), 1830 (2005)

Liu H.H, Doughty, C., Bodvarsson, G.S.: An active fracture model for unsaturated flow and transport in fractured rocks. Water Resources Research 34, 2633-2646 (1998)

Liu H H, Zhang, G., Bodvarsson, G.S.: The active fracture model: Its relation to fractal flow behavior and a further evaluation using field observations. Vadose Zone Journal 2, 259-269 (2003)

Molnar, P., Ramirez, A.: An analysis of energy expenditure in Goodwin Creek. Water Resour. Res. 34(7), 1819-1829 (1998)

Nieven, R.K.: Minimization of a free-energy-like potential for non-equilibrium flow systems at steady state. Phil. Trans. R. Soc. B2010 365, 1213-1331. doi: 10.1098/rstb.2009.0296 (2010) 
Pike, $\quad$ R.W.: $\quad$ Optimization of engineering systems, http://www.mpri.lsu.edu/bookindex.html (2001)

Rinaldo A., Banavar, J.R., Maritan, A., Trees, networks and hydrology. Water Resour. Res. 42, W06D07, doi:10.1029/2005WR004108 (2006)

Rinaldo, A., Rodriguez-Iturbe, I., Rigon, A., Bras, R.L., Ijjasz-vazquez, E., Marani, A.: Minimum energy and fractal structures of drainage networks. Water Resour. Res. 28, 2183-2191 (1992)

Rodriguez-Iturbe, I., Rinaldo, A., Rigon, A., Bras, R. L., Marani, A., Hijas-Vasquez, E.: Energy dissipation, runoff production and the three-dimensional structure of river basins. Water Resour. Res. 28(4), 1095-1103 (1992)

Sheng F, Wang, K., Zhang, R.D., Liu, H.H.: Characterizing soil preferential flow using iodine-starch staining experiments and the active region model. J Hydrol. 367(1-2), 115-124 (2009)

Wang, Z., Feyen, J., Elrick, D.E.: Prediction of fingering in porous media. Water Resour. Res. 34, 2183-2190 (1998)

Weinstock R.: Calculus of variations. Dover Publication, Inc., New York (1974) 


\section{DISCLAIMER}

This document was prepared as an account of work sponsored by the United States Government. While this document is believed to contain correct information, neither the United States Government nor any agency thereof, nor The Regents of the University of California, nor any of their employees, makes any warranty, express or implied, or assumes any legal responsibility for the accuracy, completeness, or usefulness of any information, apparatus, product, or process disclosed, or represents that its use would not infringe privately owned rights. Reference herein to any specific commercial product, process, or service by its trade name, trademark, manufacturer, or otherwise, does not necessarily constitute or imply its endorsement, recommendation, or favoring by the United States Government or any agency thereof, or The Regents of the University of California. The views and opinions of authors expressed herein do not necessarily state or reflect those of the United States Government or any agency thereof or The Regents of the University of California.

Ernest Orlando Lawrence Berkeley National Laboratory is an equal opportunity employer. 